\title{
ON THE NUMBER OF SEPARABLE LOCALLY CONVEX SPACES
}

\author{
LECH DREWNOWSKI AND ROBERT H. LOHMAN
}

\begin{abstract}
The number of distinct separable locally convex spaces is shown to be $2^{2^{c}}$. The number of distinct separable and complete, or metrizable, or normed locally convex spaces is shown to be $2^{c}$. There is no separable locally convex space that is quotient-universal for the class of separable locally convex spaces.
\end{abstract}

1. Introduction. Throughout this paper we consider only Hausdorff locally convex spaces over the fixed field $K$ of either real or complex numbers. Two locally convex spaces $E$ and $F$ are called isomorphic, denoted by $E \cong F$, if there exists an isomorphism (i.e., a linear homeomorphism) mapping $E$ onto $F$.

Let $\mathcal{L}$ be a class of locally convex spaces. A space $E$ belonging to $\mathcal{L}$ is subspace universal (respectively, quotient universal) in $\mathcal{L}$ if for each $L$ in $\mathcal{L}$ there exists a subspace $F$ of $E$ such that $L \cong F$ (respectively, $L \cong E / F$ ). In order to shorten our formulations, we denote by $\delta$ the class of all separable locally convex spaces, and by $\mathcal{C}, \mathfrak{T}, \mathscr{F}, \mathscr{T}$, and $\mathscr{B}$ the classes of all complete, metrizable, metrizable complete (Fréchet), normed, and normed complete (Banach) spaces in $\delta$, respectively.

First, let us recall a few known facts. By the classical results of Banach and Mazur [1, p. 185], [2], the spaces $C=C[0,1]$ and $l_{1}$ are, respectively, subspace universal in $\mathscr{R}$ and quotient universal in $\mathscr{B}$. Mazur and Orlicz $[9$, p. 14] proved that the Fréchet space $C(R)$ of all continuous functions $f: R \rightarrow K$, endowed with the topology of uniform convergence on compact sets, or, more abstractly, the product $C^{\aleph_{0}}$ of countably many copies of $C$, is subspace universal in T. More recently, it was observed in [7] that the product $C^{c}$ of $c$ (continuum) copies of $C$ serves as a subspace universal space in $\delta$.

It is unknown whether the classes $\delta, \mathcal{C}, \mathfrak{T}, \mathscr{T}$, or $\mathscr{F}$ possess a quotient universal space (cf. [11, p. 47]). We show that the answer is negative (Corollary 1) in the cases of $\delta, \Re$, and $\Re$. This follows as an easy consequence of the main result:

THEOREM. $\delta$ contains a subset 9 consisting of $2^{2^{c}}$ mutually nonisomorphic spaces such that each member of $\delta$ is isomorphic to a member of 9 . The same assertion holds for $\mathcal{C}, \Re$, and $\Re$ with the cardinality of 9 changed to $2^{c}$.

In addition to yielding the number of "distinct" separable locally convex spaces, the method of proof of the Theorem also shows that every subspace of

Received by the editors October 17, 1975.

AMS (MOS) subject classifications (1970). Primary 46A05.

Key words and phrases. Locally convex space, separable space, quotient-universal space.

(1) American Mathematical Society 1976 
finite codimension of a separable locally convex space is separable (Corollary 3). An example shows this is not true for subspaces of countable codimension.

\section{The main results.}

Proof of Theorem. Let $E=(E, t)$ be a locally convex space, $E^{\prime}=(E, t)^{\prime}$ its topological dual, and $E^{*}$ its algebraic dual. For each $f$ in $E^{*}$, let $t_{f}$ denote the weakest (locally convex) linear topology on $E$ such that $t \leqslant t_{f}$ and $f$ is continuous under $t_{f}$. Thus, if $E_{f}^{\prime}=\operatorname{sp}\left(E^{\prime} \cup\{f\}\right)$, then $t_{f}=\sup \left\{t, \sigma\left(E, E_{f}^{\prime}\right)\right\}$, so that $E_{f}^{\prime}=\left(E, t_{f}\right)^{\prime}$.

By definition $t \leqslant t_{f}$, and the equality holds iff $f \in E^{\prime}$. Note also that on $f^{-1}(0)$ the topologies $t$ and $t_{f}$ coincide, and since $f^{-1}(0)$ is a closed hyperplane in $\left(E, t_{f}\right)$, it follows that

$$
\left(E, t_{f}\right) \cong f^{-1}(0) \oplus K,
$$

where $f^{-1}(0)$ is endowed with the topology induced by $t$.

If $f, g \in E^{*}$, then $g \in E_{f}^{\prime}$ iff $E_{g}^{\prime} \subset E_{f}^{\prime}$ and $E_{g}^{\prime} \subset E_{f}^{\prime}$ iff $t_{g} \leqslant t_{f}$. Since $E_{f}^{\prime}=E^{\prime}$ or $E^{\prime}$ is of codimension one in $E_{f}^{\prime}, E_{g}^{\prime} \subset E_{f}^{\prime}$ holds iff $E_{g}^{\prime}=E^{\prime}$ or $E_{g}^{\prime}$ $=E_{f}^{\prime}$. Hence $t_{g} \leqslant t_{f}$ iff $t_{g}=t$ or $t_{g}=t_{f}$. Therefore, if $g \notin E^{\prime}$, then $t_{g} \leqslant t_{f}$ iff $g \in E_{f}^{\prime} \backslash E^{\prime}$. Writing $f \sim g$ when $t_{f}=t_{g}$, we define an equivalence relation on $E^{*}$; let $[f]_{\sim}=\left\{g \in E^{*}: f \sim g\right\}$. Then $[f]_{\sim}=E^{\prime}$ for each $f \in E^{\prime},[f]_{\sim}$ $=E_{f}^{\prime} \backslash E^{\prime}$ for each $f \in E^{*} \backslash E^{\prime}$, and card $[f]_{\sim}=$ card $E^{\prime}$ for each $f \in E^{*}$.

From now on let us assume that $(E, t)$ is separable. Then, for each $f \in E^{*}$, the space $\left(E, t_{f}\right)$ is separable, too. In fact, let $D$ be a countable dense subset of $E$, and suppose $D$ is not dense in $\left(E, t_{f}\right)$. Let $L$ be the $t_{f}$ closed linear subspace of $E$ spanned by $D$. By assumption, $L \neq E$. Hence there exists a nonzero $g$ in $E_{f}^{\prime}$ such that $g(L)=\{0\}$. Since $g \notin E^{\prime}$, we have $t_{g}=t_{f}$, and so by (1)

$$
\left(E, t_{f}\right)=\left(E, t_{g}\right) \cong g^{-1}(0) \oplus K .
$$

But $g^{-1}(0)$, in the topology induced by $t$, is evidently separable, hence $\left(E, t_{f}\right)$ is separable as well.

Assume $\operatorname{dim} E=m$, where $m=c$ or $m=2^{c}$. Then card $E^{*}=\operatorname{dim} E^{*}$ $=2^{m}$ and card $E^{\prime}=c$ because $E$ is separable. From each equivalence class [ ] $\neq E^{\prime}$ considered above choose an element $f$, and let $F$ denote the set thus obtained. (Instead, we may take any Hamel basis $B$ of $E^{*} / E^{\prime}$ and then define $F$ to be a set of representatives chosen out of each member of $B$.) Evidently card $F=2^{m}$, because card $[f]_{\sim}=c$ for each $f \in E^{*}$. Moreover, $F$ has the property

$$
f, g \in F \text { and } f \neq g \text { imply } t_{f} \text { and } t_{g} \text { are totally incomparable; }
$$

that is, neither $t_{f} \leqslant t_{g}$ nor $t_{g} \leqslant t_{f}$ holds.

Now, given an $f$ in $F$, we claim that $\left(E, t_{f}\right)$ can be isomorphic to $\left(E, t_{g}\right)$ for at most $m$ members $g$ of $F$. In order to see this, let $D$ be a countable dense subset of $\left(E, t_{f}\right)$. If, for some $g \in F, A$ is an isomorphism of $\left(E, t_{f}\right)$ onto $\left(E, t_{g}\right)$, then $A$ is completely determined by the values it assumes on $D$. Hence there are at most $m^{\aleph_{0}}=m$ distinct mappings $A$ of $E$ into $E$ such that for some $g \in F$ (depending on $A$ ) $A$ establishes the isomorphism of $\left(E, t_{f}\right)$ and $\left(E, t_{g}\right)$. It is not excluded, however, that different $g$ 's may correspond to the same $A$. 
So suppose $g, h \in F, g \neq h$, and $A$ is an isomorphism of $\left(E, t_{f}\right)$ onto $\left(E, t_{g}\right)$ and onto $\left(E, t_{h}\right)$. Then the identity $A A^{-1}$ is an isomorphism of $\left(E, t_{g}\right)$ onto $\left(E, t_{h}\right)$, and so $t_{g}=t_{h}$, which contradicts property (2) of $F$.

Now, for any $f, g \in F$, let $f \approx g$ mean that $\left(E, t_{f}\right)$ and $\left(E, t_{g}\right)$ are isomorphic. By what was just shown, each equivalence class $[f]_{\approx}$ contains at most $m$ members of $F$, and since card $F=2^{m}$, there are precisely $2^{m}$ distinct $\approx$ equivalence classes. Thus there exists a subset $G$ of $F$ with card $G=2^{m}$ such that the separable locally convex spaces $\left(E, t_{g}\right), g \in G$, are mutually nonisomorphic. These spaces are metrizable or normed if such is the space $E$.

Now specifying $E$ to be $C^{c}$, we have $\operatorname{dim} C^{c}=2^{c}$. The universality of $E$ guarantees the existence of a maximal set 9 of mutually nonisomorphic linear subspaces of $E$ such that each member of $\delta$ is isomorphic to a member of 9 . Clearly, we must have card $g \leqslant 2^{2^{c}}$. On the other hand, each space $\left(E, t_{g}\right), g$ $\in G$, is isomorphic to a member of 9 so that card $9 \geqslant 2^{2^{c}}$.

In the case of $\Re$ and $\Re$, let $E$ be $C^{\aleph_{0}}$ and $C$, respectively, and note that $\operatorname{dim} C^{\aleph_{0}}=\operatorname{dim} C=c$. In either case, let 9 be defined in a similar manner as before. Then card $G \leqslant 2^{c}$ and a similar argument as before shows card $q$ $\geqslant 2^{c}$.

In the case of $\mathcal{C}$, let $E$ be $C^{c}$ and choose $\mathscr{G}$ to be a maximal set of mutually nonisomorphic closed linear subspaces of $E$. Since each closed linear subspace of $E$ is an intersection of closed hyperplanes and card $E^{\prime}=c$, we have card $g \leqslant 2^{c}$. On the other hand, for each nonempty subset $P$ of $[1, \infty)$, let $l_{P}$ denote the product $\prod_{p \in P} l_{p}$. Then $l_{P}$ is complete and separable by the HewittMarczewski-Pondiczery theorem (cf. [5, p. 77], [10]). If $P, Q \subset[1, \infty)$ and $P$ $\neq Q$, then $l_{P}$ and $l_{Q}$ are not isomorphic. Indeed, suppose $P \nsubseteq Q$ and let $p$ $\in P \backslash Q$. If $A$ is an isomorphism of $l_{P}$ onto $l_{Q}$, then its restriction to the factor space $l_{p}$ is an embedding of $l_{p}$ into $l_{Q}$. It follows from Proposition 3 of [3] that there exist $q_{1}, \ldots, q_{n} \in Q$ such that $\prod_{i=1}^{n} l_{q_{i}}$ contains an isomorphic copy $X$ of $l_{p}$. As $l_{p}$ and $l_{q_{i}}$ are well known to have no infinite-dimensional isomorphic subspaces, each coordinate projection of $\prod_{i=1}^{n} l_{q_{i}}$ onto $X$ is strictly singular. However, the sum of strictly singular maps is strictly singular (cf. [6, p. 86]) so that the identity mapping on $X$ is strictly singular. The contradiction shows that $l_{P}$ and $l_{Q}$ are not isomorphic. Since $\mathscr{9}$ must contain an isomorphic copy of $l_{P}$ for $\varnothing \neq \stackrel{P}{P} \subset[1, \infty)$, card $q \geqslant 2^{c}$.

REMARK. It is well known that a set $g$ satisfying the statement of the Theorem can be chosen for the classes $\mathscr{F}$ and $\mathscr{B}$ with card $\mathscr{G}=c$.

COROLlary 1. There is no quotient-universal space in the classes $\mathscr{T}, \mathfrak{T}$, and $\delta$.

Proof. Consider, for instance, the case of $\delta$. Suppose that a space $E \in \delta$ is quotient-universal in $\delta$. Then $E$ admits at most $2^{c}$ distinct (up to isomorphism) Hausdorff quotients, because card $E^{\prime}=c$ and each closed linear subspace of $E$ is an intersection of a family of closed hyperplanes $f^{-1}(0), f \in E^{\prime}$. But there are $2^{2 c}$ mutually nonisomorphic separable locally convex spaces, yielding a contradiction. The proof in the cases of $\Re$ and $\Re$ is similar.

By a result of Dieudonné [4], every finite-codimensional subspace of a barrelled space is barrelled (and hence a Mackey space). The subspaceuniversal spaces $E$ used in the proof of the Theorem are barrelled as products of barrelled spaces, and the spaces $\left(E, t_{f}\right)$ are barrelled since they are 
isomorphic to products of one-codimensional subspaces of $E$ with $K$. Hence we have

COROLlaRY 2. There are precisely $2^{2^{c}}, 2^{c}$ and $2^{c}$ distinct barrelled (resp. Mackey) spaces in $\delta$, $\Re$, and $\Re$, respectively.

Our next result is also an easy consequence of the proof of the Theorem (cf. [14]).

COROllaRY 3. Every subspace of finite codimension of a separable locally convex space is separable.

REMARK. In view of some recent results showing that certain properties of locally convex spaces are inherited by subspaces of countable codimension (see e.g. [12] and [13] for the property of being barrelled), one might ask whether, in Corollary 3, "finite" can be replaced by "countable". We give a counterexample showing that this need not be the case. Note that there exists a separable locally convex space $E$ with a closed nonseparable subspace $F$. An example of such a space was given in [8]. Let $S$ be a countable subset of $E$ whose closed linear span is all of $E$. Then $G=\operatorname{sp}(F \cup S)$, in the relative topology, is a separable locally convex space whose countable-codimensional closed subspace $F$ is not separable.

\section{REFERENCES}

1. S. Banach, Théorie des opérations linéaires, Mongrafie Mat., PWN, Warsaw, 1932; reprint, Chelsea, New York, 1955. MR 17, 175.

2. S. Banach and S. Mazur, Zur Theorie der linearen Dimension, Studia Math. 4 (1933), $100-112$.

3. J. Diestel and R. H. Lohman, Applications of mapping theorems to Schwartz spaces and projections, Michigan Math. J. 20 (1973), 39-44. MR 47 \#5541.

4. J. Dieudonné, Sur les propriétés de permanence de certains espaces vectoriels topologiques, Ann. Soc. Polon. Math. 25 (1952), 50-55. MR 15, 38.

5. R. Engelking, Outline of general topology, Wiley, New York, 1968.

6. S. Goldberg, Unbounded linear operators: Theory and applications, McGraw-Hill, New York, 1966. MR 34 \#80.

7. R. H. Lohman, An embedding theorem for separable locally convex spaces, Canad. Math. Bull. 14 (1971), 119-120.

8. R. H. Lohman and W. J. Stiles, On separability in linear topological spaces, Proc. Amer. Math. Soc. 42 (1974), 236-237. MR 48 \# 4694.

9. S. Mazur and W. Orlicz, Sur les espaces métriques linéaires. II, Studia Math. 13 (1953), 137-179. MR 16, 932.

10. E. S. Pondiczery, Power problems in abstract spaces, Duke Math. J. 11 (1944), 835-837. MR 6, 119.

11. S. Rolewicz, Metric linear spaces, Polish Scientific Publishers, Warsaw, 1972.

12. S. Saxon and M. Levin, Every countable-codimensional subspace of a barrelled space is barrelled, Proc. Amer. Math. Soc. 29 (1971), 91-96. MR 43 \#6691.

13. M. Valdivia, Absolutely convex sets in barrelled spaces, Ann. Inst. Fourier (Grenoble) 21 (1971), fasc. 2, 3-13. MR 48 \#11968.

14. - On weak compactness, Studia Math. 49 (1973), 35-40. MR 48 \# 11969.

Institute of Mathematics, A. Mickiewicz University, Ul. MAtejki 48/49, 60-769 Poznań, Poland 